\title{
Correction to: Investigation of organic material self-heating in oxygen-depleted condition within a coal-waste dump in Upper Silesia Coal Basin, Poland
}

\author{
Ádám Nádudvari ${ }^{1}$ - Monika J. Fabiańska ${ }^{1}$ - Magdalena Misz-Kennan ${ }^{1} \cdot$ Justyna Ciesielczuk ${ }^{1} \cdot$ Adam Kowalski $^{2}$
}

Published online: 13 January 2020

(C) Springer-Verlag GmbH Germany, part of Springer Nature 2020

\section{Correction to: Environmental Science and Pollution Research} https://doi.org/10.1007/s11356-019-07336-8

The original publication of this paper contains a mistake.

The correct caption of figures 7 and 8 is shown in this paper.

The original article was corrected.

Fig. 7 Schematic diagram of the transformation (aromatization) process of alkylcyclohexane during self-heating a<smiles>CCCCCCCCCC1CCCCC1</smiles>

b<smiles>CCCCCCCCCC1CCCCC1[AlH]CCCCCOCCOCCO</smiles><smiles>CCCCCCCCCc1ccccc1</smiles>

$T$<smiles>CCCCCCCCCc1ccccc1C</smiles>
alkylmethylbenzene

The online version of the original article can be found at https://doi.org/ 10.1007/s11356-019-07336-8

Ádám Nádudvari

adam.nadudvari@us.edu.pl

1 Faculty of Natural Sciences, University of Silesia, 60 Będzińska Street, 41-200 Sosnowiec, Poland

2 Faculty of Geology, Geophysics and Environmental Protection, Akademia Górniczo-Hutnicza (AGH) University of Science andTechnology, 30 Mickiewicza Avenue, 30-059 Krakow, Poland 

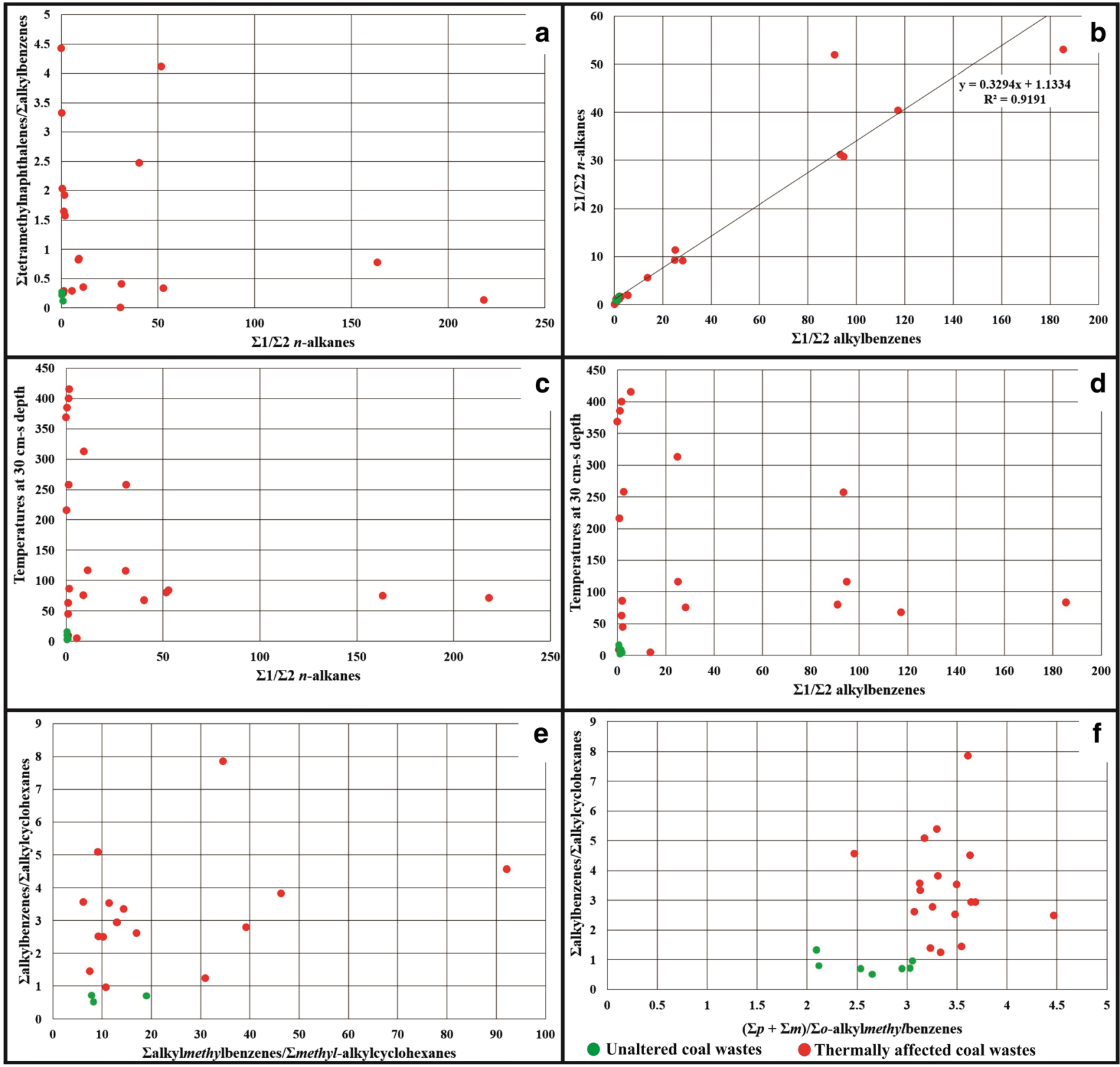

Fig. 8 Various ratios combined with temperature measurements at $30 \mathrm{~cm}$ depth to distinguish heated- and unaltered coal waste. $\Sigma 1 / \Sigma 2 n$-alkanes: short chain $\left(n-\mathrm{C}_{13}-\mathrm{C}_{22}\right) /$ long chain $\left(\mathrm{n}-\mathrm{C}_{23}-\mathrm{C}_{36}\right) \Sigma 1 / \Sigma 2$ alkylbenzenes:short chain $\left(\mathrm{C}_{12}-\mathrm{C}_{21}\right) /$ long chain $\left(\mathrm{C}_{22}-\mathrm{C}_{32}\right)$ Etetramethylnaphthalenes: 1,3,6,7-tetramethylnaphthalene; 1,2,4,6+1,

tetramethylnaphthalene; 2,3,6,7-tetramethylnaphthalene; 1,2,6,7tetramethylnaphthalene; 1,2,3,7- tetramethylnaphthalene; 1,2,3,6tetramethylnaphthalene; 1,2,5,6 -+ 1,2,3,5-tetramethylnaphthalene Ealkylmethylbenzenes: $\left(\mathrm{C}_{12}-\mathrm{C}_{31}\right)$ Emethylalkylcyclohexanes: $\left(\mathrm{C}_{15}-\mathrm{C}_{29}\right)$ Ealkylcylohexanes: $\left(\mathrm{C}_{13}-\mathrm{C}_{33}\right)(\Sigma \mathrm{p}+\Sigma \mathrm{m}) / \Sigma \mathrm{No}$ alkylmethylbenzenes: $\left(\Sigma\right.$ paraC $_{12}-\mathrm{C}_{31}+\Sigma$ metaC $\left._{12}-\mathrm{C}_{31}\right) / \Sigma$ orthoC ${ }_{12}-\mathrm{C}_{31}$ alkylmethylbenzenes 